\title{
BESSEL AND FLETT POTENTIALS ASSOCIATED WITH DUNKL OPERATORS ON $\mathbb{R}^{d *}$
}

\author{
NÉJIB BEN SALEM ${ }^{\dagger}$, ANIS EL GARNA ${ }^{\dagger}$, AND SAMIR KALLEL $^{\dagger}$
}

\begin{abstract}
Analogous of Bessel and Flett potentials are defined and studied for the Dunkl transform associated with a family of weighted functions that are invariant under a reflection group. We show that the Dunkl-Bessel potentials, of positive order, can be represented by an integral involving the $k$-heat transform and we give some applications of this result.

Also, we obtain an explicit inversion formula for the Dunkl-Flett potentials, which are interpreted as negative fractional powers of a certain operator expressed in terms of the Dunkl-Laplacian.
\end{abstract} tial.

Key words. Dunkl operator, Poisson transform, heat transform, Bessel potential, Flett poten-

AMS subject classifications. $32 \mathrm{~A} 55,47 \mathrm{H} 5 \mathrm{O}, 31 \mathrm{~A} 1 \mathrm{O}$

Introduction. In this paper we consider the harmonic analysis associated with differential-difference operators $\mathcal{D}_{j}, j=1, \cdots, d$, on $\mathbb{R}^{d}$ introduced by Dunkl [4] and called in the literature Dunkl operators associated with some finite reflection groups. These operators play an important role in pure Mathematics and Physics.

For a family of weighted functions $h_{k}$, invariant under a reflection group, Dunkl transform $\mathcal{F}_{k}$ is an extension of the ordinary Fourier transform which is an isometry of $L^{2}\left(\mathbb{R}^{d}, h_{k}^{2}\right)$, the space of squared integrable functions with respect to the measure $h_{k}^{2}(x) d x$ (see $\left.[6]\right) . \mathcal{F}_{k}$ is defined by

$$
\mathcal{F}_{k}(f)(x)=c_{h} \int_{\mathbb{R}^{d}} E_{k}(x,-i y) f(y) h_{k}^{2}(y) d y, f \in L^{1}\left(\mathbb{R}^{d}, h_{k}^{2}\right),
$$

where the usual character $e^{-i<x, y>}$ is replaced by $E_{k}(x,-i y)=V_{k}\left(e^{-i<x, .>}\right)(y), V_{k}$ is a positive linear operator and $c_{h}$ is a constant. Dunkl transform permits to introduce the Dunkl translation operator, $\mathcal{T}_{y}^{k}, y \in \mathbb{R}^{d}$, on $L^{2}\left(\mathbb{R}^{d}, h_{k}^{2}\right)$ defined by

$$
\mathcal{F}_{k}\left(\mathcal{T}_{y}^{k} f\right)(x)=E_{k}(y,-i x) \mathcal{F}_{k}(f)(x), \quad x \in \mathbb{R}^{d} \text {, and } f \in L^{2}\left(\mathbb{R}^{d}, h_{k}^{2}\right) .
$$

The explicit expression of $\mathcal{T}_{y}^{k} f$ is known only in some special cases and it is not a positive operator in general. Also, it generates a new convolution product structure associated with Dunkl operators denoted here by $*_{k}$.

In this work, we study the operator $G_{t}^{k}, t>0$, called the k-heat transform, defined on $L^{p}\left(\mathbb{R}^{d}, h_{k}^{2}\right), p \in[1,+\infty]$, by $G_{t}^{k} f=F_{t}^{k} *_{k} f, t>0$, where $F_{t}^{k}$ is a solution of the heat-equation for the $k$-Laplacian $\triangle_{k}=\sum_{j=1}^{d} \mathcal{D}_{j}^{2}$. And we give some of its properties. Next, as in the ordinary case, we define the Dunkl-Bessel potential $\mathcal{J}_{\alpha}^{k}$, of positive order $\alpha$, of a sufficiently smooth function via the Dunkl transform as $\mathcal{F}_{k}\left(\mathcal{J}_{\alpha}^{k} f\right)(x)=$ $\left(1+\|x\|^{2}\right)^{-\frac{\alpha}{2}} \mathcal{F}_{k}(f)(x)$. To be more precise, $\mathcal{J}_{\alpha}^{k}$ can be written as a convolution operator given by

$$
\mathcal{J}_{\alpha}^{k} f=b_{\alpha}^{k} *_{k} f, \text { where } \mathcal{F}_{k}\left(b_{\alpha}^{k}\right)(x)=\left(1+\|x\|^{2}\right)^{-\frac{\alpha}{2}} .
$$

\footnotetext{
*Received May 6, 2008; accepted for publication November 27, 2008.

${ }^{\dagger}$ Department of Mathematics, Faculty of Sciences of Tunis, Campus Universitaire, 2092 Tunis,

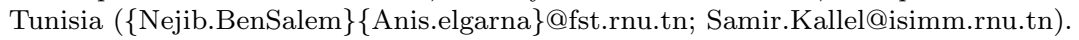


We show that when $f \in L^{p}\left(\mathbb{R}^{d}, h_{k}^{2}\right), \mathcal{J}_{\alpha}^{k} f$ can be represented by an integral of a very simple form involving the $k$-heat transform of $f$. This representation permits to extend the definition of the Dunkl-Bessel potential of ordre $\alpha$ first to a class of $k$-temperatures i.e. solutions of the heat equation for the $k$-Laplacian $\triangle_{k}$ on the upper-half space $\mathbb{R}^{d} \times \mathbb{R}_{+}$, then to the space of tempered distributions on $\mathbb{R}^{d}$.

The Dunkl-Bessel potential is closely related to the Dunkl-Riesz potential (see [15]), since the kernel of the Dunkl-Bessel potential has essentially the same local behaviour as that of the Dunkl-Riesz potential as $\|x\| \rightarrow 0$, but the behaviour of the kernel of the Dunkl-Riesz potential at infinity is not as good as that of the Dunkl-Bessel potential. There are, however, other fractional integral operators whose behaviours are roughly midway between the Dunkl-Riesz and the Dunkl-Bessel potentials, for instance, the Dunkl-Flett potentials $\mathcal{I}_{k}^{\alpha}$ of positive ordre $\alpha$, which is studied in the last section and given by $\mathcal{I}_{k}^{\alpha}=\left(I-\triangle_{k}^{\frac{1}{2}}\right)^{-\alpha}$. It has the following integral representation $\mathcal{I}_{k}^{\alpha} f(x)=\frac{1}{\Gamma(\alpha)} \int_{0}^{+\infty} t^{\alpha-1} e^{-t} P_{t}^{k} f(x) d t$, where $P_{t}^{k}$ is the Poisson transform associated with Dunkl operators on $\mathbb{R}^{d}$. $\mathcal{I}_{k}^{\alpha}$ generalizes the usual Flett potential (which corresponds to $k=0$ ) introduced by Flett in [8].

One important problem concerning the Dunkl-Flett potential is obtaining an explicit inversion formula. For this aim, we introduce an integral transform

$$
\mathcal{W}_{t}^{k, \mu} f(x)=\int_{\mathbb{R}_{+}} e^{-t \eta} P_{t \eta}^{k} f(x) d \mu(\eta), x \in \mathbb{R}^{d}, f \in L^{p}\left(\mathbb{R}^{d}, h_{k}^{2}\right), t>0 .
$$

$\mathcal{W}_{t}^{k, \mu}$ is called the $k$-weighted wavelet-like transform of $f$ associated with the $k$-Poisson transform and generated by an appropriate Borel measure $\mu$ on $\mathbb{R}_{+}$according to our needs. The explicit inversion formula for the Dunkl-Flett potential is obtained in term of the $\mathcal{W}_{t}^{k, \mu}$ transform.

The paper is arranged as follows. In the first section we collect some background materials for the harmonic analysis associated with Dunkl operators. In the second section we give some properties of the $k$-heat transform of a measurable function. In the third section we study the Dunkl-Bessel potential $\mathcal{J}_{\alpha}^{k} f$ of a function $f \in$ $L^{p}\left(\mathbb{R}^{d}, h_{k}^{2}\right)$ of positive order $\alpha$ and we show that $\mathcal{J}_{\alpha}^{k} f$ can be represented by an integral involving the $k$-heat transform of $f$. By making use of this representation we extend the definition of the Dunkl-Bessel potential of order $\alpha$ to a class of $k$-temperatures. We finish this section by studing the Dunkl-Bessel potential of a temperated distribution. In the last section we define the Dunkl-Flett potential and we obtain the explicit inversion formula.

1. Preliminaries. The purpose of this section is to establish our basic notations and collect some further facts on Dunkl transform, Dunkl translation operator and Dunkl convolution product which serve as fundamental tools for our later investigations. General references here are $[4,5,6],[7],[11],[14]$ and [16].

\section{Notations:}

- $C_{0}\left(\mathbb{R}^{d}\right)$ the space of continuous functions vanishing at infinity, provided with the usual topology of uniform convergence on $\mathbb{R}^{d}$.

- $\mathcal{E}\left(\mathbb{R}^{d}\right)$ the space of $C^{\infty}$-functions on $\mathbb{R}^{d}$, endowed with the usual topology of uniform convergence of the functions and their derivatives of all order on compact subsets of $\mathbb{R}^{d}$. 
- $S\left(\mathbb{R}^{d}\right)$ the space of $C^{\infty}$-functions on $\mathbb{R}^{d}$ which are rapidly decreasing as their derivatives, provided with the topology defined by the seminorms

$$
\rho_{s, l}(\varphi)=\sup _{x \in \mathbb{R}^{d},|\nu| \leq s}\left(1+\|x\|^{2}\right)^{l}\left|\mathcal{D}^{\nu} \varphi(x)\right|, s, l \in \mathbb{N},
$$

with $\mathcal{D}^{\nu}=\mathcal{D}_{1}^{\nu_{1}} o \cdots o \mathcal{D}_{d}^{\nu_{d}}, \nu=\left(\nu_{1}, \cdots, \nu_{d}\right) \in \mathbb{N}^{d}$.

- $S^{\prime}\left(\mathbb{R}^{d}\right)$ the space of tempered distributions on $\mathbb{R}^{d}$, it is the topological dual of $S\left(\mathbb{R}^{d}\right)$

Let $G$ be a finite reflection group (also called Coxeter group) on $\mathbb{R}^{d}$ associated with a root system $\mathcal{R}$ and $\mathcal{R}_{+}$the positive subsystem of $\mathcal{R}$ ([5] ,[7], [10]). We denote by $k$ a nonnegative multiplicity function defined on $\mathcal{R}$ with the property that $k$ is $G$-invariant. We associate with $k$ the weight function

$$
h_{k}(x)=\prod_{v \in \mathcal{R}_{+}}|<x, v>|^{k(v)}, x \in \mathbb{R}^{d} .
$$

The Dunkl operators associated with $G$ and $k$ are given by [4]

$$
\mathcal{D}_{i} f(x)=\partial_{i} f(x)+\sum_{v \in \mathcal{R}_{+}} k(v) \frac{f(x)-f\left(\sigma_{v}(x)\right)}{<x, v>}<v, e_{i}>, 1 \leq i \leq d, x \in \mathbb{R}^{d},
$$

where $\partial_{i}$ is the ordinary partial derivative with respect to $x_{i}$ and $e_{1}, \cdots, e_{d}$ are the standard unit vectors of $\mathbb{R}^{d}$. The above definition does not depend on the special choice of $\mathcal{R}_{+}$, thanks to the $G$ invariance of $k$. In the case $k=0$, the $\mathcal{D}_{i}, i=1, \cdots d$, reduce to the corresponding partial derivatives. These operators map $\mathcal{P}_{n}^{d}$ to $\mathcal{P}_{n-1}^{d}$, where $\mathcal{P}_{n}^{d}$ is the space of homogeneous polynomials of degree $n$ in $d$ variables. More importantly, these operators mutually commute ; that is, $\mathcal{D}_{i} \mathcal{D}_{j}=\mathcal{D}_{j} \mathcal{D}_{i}$. The Dunkl Laplacian is defined by $\triangle_{k}=\sum_{i=1}^{d} \mathcal{D}_{i}^{2}$.

There is a linear isomorphism that intertwines the algebra generated by Dunkl's operators with the algebra of partial differential operators. The intertwining operator $V_{k}$ is an operator determined uniquely by

$$
V_{k} \mathcal{P}_{n}^{d} \subset \mathcal{P}_{n}^{d}, \quad V_{k} 1=1, \quad \mathcal{D}_{i} V_{k}=V_{k} \partial_{i}, \quad 1 \leq i \leq d .
$$

The explicit formula of $V_{k}$ is not known in general. In [10], it was shown that $V_{k}$ has a Laplace-type representation as follows: For every $x \in \mathbb{R}^{d}$, there exists a unique probability measure $\mu_{x}^{k}$ on the Borel $\sigma$-algebra of $\mathbb{R}^{d}$ such that

$$
V_{k} p(x)=\int_{\mathbb{R}^{d}} p(\xi) d \mu_{x}^{k}(\xi),
$$

for each polynomial function $p$ on $\mathbb{R}^{d}$. The representing measures $\mu_{x}^{k}$ are compactly supported with $\operatorname{supp}_{x}^{k} \subseteq C(x):=c o\{v x, v \in G\}$, the convex hull of the $G$-orbit of $x$ in $\mathbb{R}^{d}$.

By means of formula (1), $V_{k}$ may be extended to various larger function spaces, including $\mathcal{E}\left(\mathbb{R}^{d}\right)$. We denote this extension by $V_{k}$ again. In fact, $V_{k}$ establishes a topological isomorphism from $\mathcal{E}\left(\mathbb{R}^{d}\right)$ onto itself (see [16] and [3]). For $y \in \mathbb{R}^{d}$ the function

$$
E_{k}(x, y)=V_{k}\left(e^{<\cdot, y>}\right)(x)=\int_{\mathbb{R}^{d}} e^{<\xi, y>} d \mu_{x}^{k}(\xi), x \in \mathbb{R}^{d}
$$


is called the Dunkl kernel and generalizes the usual exponential function $e^{<x, y>}$. It plays an important role in the development of the Dunkl transform which is an integral transform generalizing the ordinary Fourier transform.

The Dunkl kernel possesses the following properties: for $x, y \in \mathbb{R}^{d}$, we have

$$
\begin{gathered}
E_{k}(x, y)=E_{k}(y, x), E_{k}(x, 0)=1, E_{k}(-i x, y)=\overline{E_{k}(i x, y)} \\
\left|E_{k}(i x, y)\right| \leq 1 \text { and } E_{k}(\lambda x, y)=E_{k}(x, \lambda y), \lambda \in \mathbb{C} .
\end{gathered}
$$

For each $\nu \in \mathbb{N}^{d}$ there exists a constant $d_{\nu}>0$ such that

$$
\left|\partial_{x}^{\nu} E_{k}(x, z)\right| \leq d_{\nu}|z|^{|\nu|} e^{|x||\mathcal{R} e z|} \text {, for all } x \in \mathbb{R}^{d}, z \in \mathbb{C}^{d},
$$

here the underscript $x$ denotes the operators act with respect to the $x$-variable. Hereafter, we denote by $L^{p}\left(\mathbb{R}^{d}, h_{k}^{2}\right), p \in[1, \infty]$, the space of mesurable functions on $\mathbb{R}^{d}$ such that

$$
\|f\|_{k, p}=\left(\int_{\mathbb{R}^{d}}|f(x)|^{p} h_{k}^{2}(x) d x\right)^{\frac{1}{p}}<+\infty, \quad 1 \leq p<\infty
$$

and

$$
\|f\|_{k, \infty}=e s s \sup _{x \in \mathbb{R}^{d}}|f(x)|<+\infty .
$$

For $f \in L^{1}\left(\mathbb{R}^{d}, h_{k}^{2}\right)$, the Dunkl transform is defined by

$$
\forall y \in \mathbb{R}^{d}, \mathcal{F}_{k}(f)(y):=c_{h} \int_{\mathbb{R}^{d}} f(x) E_{k}(x,-i y) h_{k}^{2}(x) d x,
$$

where $c_{h}^{-1}=\int_{\mathbb{R}^{d}} h_{k}^{2}(x) e^{-\|x\|^{2} / 2} d x$.

Many properties of the ordinary Fourier transform carry over the Dunkl transform. For instance, if we denote by $\mathcal{A}_{k}\left(\mathbb{R}^{d}\right)=\left\{f \in L^{1}\left(\mathbb{R}^{d}, h_{k}^{2}\right): \mathcal{F}_{k}(f) \in L^{1}\left(\mathbb{R}^{d}, h_{k}^{2}\right)\right\}$, then we have the following results (see $[6,7]$ ).

Proposition 1.1.

- For $f \in L^{1}\left(\mathbb{R}^{d}, h_{k}^{2}\right), \mathcal{F}_{k}(f)$ is in $C_{0}\left(\mathbb{R}^{d}\right)$.

- Let $f \in \mathcal{A}_{k}\left(\mathbb{R}^{d}\right)$, we have the inversion formula $f(x)=$ $\int_{\mathbb{R}^{d}} E_{k}(i x, y) \mathcal{F}_{k}(f)(y) h_{k}^{2}(y) d y$, a.e.

- The Dunkl transform extends to an isometry of $L^{2}\left(\mathbb{R}^{d}, h_{k}^{2}\right)$.

- Let $f \in S\left(\mathbb{R}^{d}\right)$, we have $\mathcal{F}_{k}\left(\mathcal{D}_{j} f\right)(y)=i y_{j} \mathcal{F}_{k}(f)(y)$.

For $y \in \mathbb{R}^{d}$, we define a translation operator $\mathcal{T}_{y}^{k}$ associated with Dunkl operators on $L^{2}\left(\mathbb{R}^{d}, h_{k}^{2}\right)$ by

$$
\mathcal{F}_{k}\left(\mathcal{T}_{y}^{k} f\right)(x)=E_{k}(y,-i x) \mathcal{F}_{k}(f)(x), x \in \mathbb{R}^{d} .
$$

It plays the role of the ordinary translation $\mathcal{T}_{y} f=f(.-y)$ of $\mathbb{R}^{d}$, since the Fourier transform of $\mathcal{T}_{y}$ is given by: $\mathcal{F}\left(\mathcal{T}_{y} f\right)(x)=e^{-i<x, y>} \mathcal{F}(f)(x)$. The generalized translation operator has been studied in $[11,14,17]$. 
Proposition 1.2. (see [11]) Let $f \in \mathcal{A}_{k}\left(\mathbb{R}^{d}\right)$ (resp. $f \in \mathcal{E}\left(\mathbb{R}^{d}\right)$ ) be radial and let $f(x)=f_{o}(\|x\|)$. Then

$$
\mathcal{T}_{y}^{k} f(x)=\int_{C(y)} f_{o}\left(\sqrt{\|x\|^{2}+\|y\|^{2}+2<x, \eta>}\right) d \mu_{y}^{k}(\eta),
$$

which gives that $\mathcal{T}_{y}^{k} f(x) \geq 0$ for all $y \in \mathbb{R}^{d}$ if $f(x)=f_{o}(\|x\|) \geq 0$.

Proposition 1.3. (see [14])

(i) If $f \in C_{0}^{\infty}\left(\mathbb{R}^{d}\right)$ with compact support, then for $1 \leq p \leq \infty, \lim _{y \rightarrow 0}\left\|\mathcal{T}_{y}^{k} f-f\right\|_{k, p}=0$.

(ii) Let $G=\mathbf{Z}_{2}^{d}$. For $f \in L^{p}\left(\mathbb{R}^{d}, h_{k}^{2}\right), 1 \leq p \leq \infty,\left\|\mathcal{T}_{y}^{k} f\right\|_{k, p} \leq 3\|f\|_{k, p}$.

REMARK. Let us mention that there is an abstract formula for $\mathcal{T}_{y}^{k}$ given in terms of the intertwining operator $V_{k}$ and its inverse, (see[16]). It takes the form of

$$
\mathcal{T}_{y}^{k} f(x)=V_{k, x} V_{k, y}\left[V_{k}^{-1}(f)(x+y)\right], \forall f \in \mathcal{E}\left(\mathbb{R}^{d}\right) .
$$

The translation operator $\mathcal{T}_{y}^{k}$ can be used to define a convolution structure on $L^{2}\left(\mathbb{R}^{d}, h_{k}^{2}\right)$, by

$$
f *_{k} g(x)=\int_{\mathbb{R}^{d}} f(y) \mathcal{T}_{-x}^{k} \check{g}(y) h_{k}^{2}(y) d y, \quad f, g \in L^{2}\left(\mathbb{R}^{d}, h_{k}^{2}\right),
$$

where $\check{g}(y)=g(-y)$. Since, $\mathcal{T}_{x}^{k} \check{g} \in L^{2}\left(\mathbb{R}^{d}, h_{k}^{2}\right)$ the convolution is well defined. This convolution structure has been considered by several authors $[11,14,17]$ and the references therein. It is associative, commutative and satisfies the following property:

$$
\mathcal{F}_{k}\left(f *_{k} g\right)=\mathcal{F}_{k}(f) \mathcal{F}_{k}(g) .
$$

For the general reflection group, the following result is proved in [14].

THEOREM 1.4. Let $g$ be a bounded radial function in $L^{1}\left(\mathbb{R}^{d}, h_{k}^{2}\right)$. Then $f *_{k} g$ initially defined in (5) on the intersection of $L^{1}\left(\mathbb{R}^{d}, h_{k}^{2}\right)$ and $L^{2}\left(\mathbb{R}^{d}, h_{k}^{2}\right)$ extends to all $L^{p}\left(\mathbb{R}^{d}, h_{k}^{2}\right), 1 \leq p \leq \infty$ as a bounded operator. In particular, $\left\|f *_{k} g\right\|_{k, p} \leq$ $\|g\|_{k, 1}\|f\|_{k, p}$.

2. The $k$-heat transforms of a function. In this section we give some properties of the $k$-heat transforms of a measurable function $f$. For $t>0$, let $F_{t}^{k}$ be the function defined by

$$
F_{t}^{k}(x)=(2 t)^{-\left(\gamma_{k}+\frac{d}{2}\right)} \exp \left(-\frac{\|x\|^{2}}{4 t}\right),
$$

which is a solution of the heat equation for the $k$-Laplacian $\triangle_{k} U(x, t)=\partial_{t} U(x, t)$, where $\triangle_{k}$ is applied to $x$ variables (see [9]). The function $F_{t}^{k}$ may be called the heat kernel associated with Dunkl operators or the k-heat kernel and it has the following basic properties. (Throughout this paper we use the convention that $B$ denotes a generic constant, depending on $d, k, \nu$ or other fixed parameters, its value may change from line to line).

Lemma 2.1. For all $\left.(x, t) \in \mathbb{R}^{d} \times\right] 0,+\infty[$, we have

(i) $0 \leq F_{t}^{k}(x) \leq(2 t)^{-\gamma_{k}-\frac{d}{2}}$.

(ii) For $\nu=\left(\nu_{1}, \cdots, \nu_{d}\right) \in \mathbb{N}^{d}$, we have

$$
\mathcal{D}^{\nu} F_{t}^{k}(x)=t^{-\frac{|\nu|}{2}} F_{t}^{k}(x) P,
$$


where $P$ is a polynomial of degree $|\nu|=\nu_{1}+\cdots+\nu_{d}$ in the variables $\frac{x_{i}}{2 \sqrt{t}}(i=1, \cdots, d)$ with coefficients depending only on $d, \nu$ and $k$.

(iii) If $m$ is a positive integer, then

$$
\partial_{t}^{m} F_{t}^{k}(x)=t^{-m} R\left(\frac{\|x\|^{2}}{4 t}\right) F_{t}^{k}(x),
$$

with $R$ is a polynomial of degree $m$ with coefficients depending only on $m, d$ and $k$.

(iv) If $t>0, \eta \geq 0$ and $g$ is measurable on $] 0,+\infty[$, then

$$
\int_{\|x\| \geq \eta} g\left(\|x\|^{2}\right) F_{t}^{k}(x) h_{k}^{2}(x) d x=\frac{1}{c_{h} \Gamma\left(\gamma_{k}+\frac{d}{2}\right)} \int_{\frac{\eta^{2}}{4 t}}^{+\infty} g(4 t \sigma) e^{-\sigma} \sigma^{\lambda_{k}} d \sigma
$$

whenever the integral on the right exists. In particular,

$c_{h} \int_{\mathbb{R}^{d}} F_{t}^{k}(x) h_{k}^{2}(x) d x=1, \int_{\mathbb{R}^{d}}\left(\frac{\|x\|^{2}}{4 t}\right)^{a} F_{t}^{k}(x) h_{k}^{2}(x) d x=\frac{\Gamma\left(\gamma_{k}+\frac{d}{2}+a\right)}{c_{h} \Gamma\left(\gamma_{k}+\frac{d}{2}\right)},\left(a>-\frac{d}{2}-\gamma_{k}\right)$.

(v) $\int_{\mathbb{R}^{d}}\left(\frac{\partial}{\partial t}\right)^{m} F_{t}^{k}(x) h_{k}^{2}(x) d x=0, m \in \mathbb{N} \backslash\{0\}$.

(vi) If $\nu \in \mathbb{N}^{d}$ and $m \in \mathbb{N} \backslash\{0\}$, then

$$
\begin{gathered}
\int_{\mathbb{R}^{d}}\left|\mathcal{D}^{\nu} F_{t}^{k}(x)\right| h_{k}^{2}(x) d x \leq B(d, k, \nu) t^{-\frac{|\nu|}{2}}, \\
\int_{\mathbb{R}^{d}}\left|\frac{\partial^{m}}{\partial t^{m}} F_{t}^{k}(x)\right| h_{k}^{2}(x) d x \leq B(d, k, m) t^{-m},
\end{gathered}
$$

where $B(d, k, \nu)$ and $B(d, k, m)$ are constants.

(vii) For all $\left.(x, t) \in \mathbb{R}^{d} \times\right] 0,+\infty\left[, \mathcal{F}_{k}\left(F_{t}^{k}\right)(x)=e^{-t\|x\|^{2}}\right.$.

(viii) For all $\left.(x, y, t) \in \mathbb{R}^{d} \times \mathbb{R}^{d} \times\right] 0,+\infty[$, we have

$$
\mathcal{T}_{-y}^{k} F_{t}^{k}(x)=\frac{1}{(2 t)^{\gamma_{k}+\frac{d}{2}}} e^{-\left(\frac{\|x\|^{2}+\|y\|^{2}}{4 t}\right)} E_{k}\left(\frac{x}{\sqrt{2 t}}, \frac{y}{\sqrt{2 t}}\right) .
$$

(ix) If $s>0, t>0$, and $x \in \mathbb{R}^{d}$, we have

$$
\int_{\mathbb{R}^{d}} F_{s}^{k}(y) \mathcal{T}_{-y}^{k} F_{t}^{k}(x) h_{k}^{2}(y) d y=F_{t+s}^{k}(x) .
$$

Proof. (i) is clear. (ii) and (iii) are obtained by a short calculation. To obtain (iv), we use the spherical-polar coordinates and a change of variables. The assertion (v) follows from representation (iv) by taking the differentiations under the integral sign. (vi) is a consequence of (ii) and (iii). Using Lemma 4.11 of [7] we deduce the assertion (vii). (viii) is a special case of Proposition 1.2. (ix) is obtained by applying Dunkl transform to $F_{s}^{k} *_{k} F_{t}^{k}$ and using (vii).

DEFINITION 2.2. The $k$-heat transform of a smooth measurable function $f$ on $\mathbb{R}^{d}$ is given by

$$
G_{t}^{k}(f)(x)=\int_{\mathbb{R}^{d}} \mathcal{T}_{-y}^{k} F_{t}^{k}(x) f(y) h_{k}^{2}(y) d y, t>0 .
$$


Since, $F_{t}^{k}, t>0$, is a bounded radial function in $L^{1}\left(\mathbb{R}^{d}, h_{k}^{2}\right)$, then for all $f \in$ $L^{p}\left(\mathbb{R}^{d}, h_{k}^{2}\right), p \in[1,+\infty], G_{t}^{k}(f), t>0$, is well defined and continuous on $\mathbb{R}^{d}$.

THEOREM 2.3. Let $f$ be a measurable bounded function on $\mathbb{R}^{d}$. Then, (i) $(x, t) \mapsto G_{t}^{k}(f)(x)$ is infinitely differentiable on $\left.\mathbb{R}^{d} \times\right] 0,+\infty[$ and it is a solution of the heat equation for the $k$-Laplacian. Further, if $\nu \in \mathbb{N}^{d}, m \in \mathbb{N}$, then for all $t>0$

$$
\mathcal{D}^{\nu} G_{t}^{k}(f)=\mathcal{D}^{\nu} F_{t}^{k} *_{k} f \text { and } \partial_{t}^{m} G_{t}^{k}(f)=\partial_{t}^{m} F_{t}^{k} *_{k} f .
$$

(ii) For all $s, t>0$ and $x \in \mathbb{R}^{d}$, we have $G_{t+s}^{k}(f)(x)=$ $\int_{\mathbb{R}^{d}} \mathcal{T}_{-y}^{k} F_{t}^{k}(x) G_{s}^{k}(f)(y) h_{k}^{2}(y) d y$.

(iii) For all $\left.(x, t) \in \mathbb{R}^{d} \times\right] 0,+\infty[$, and for all $s>t$, we have

$$
\left|G_{t}^{k}(f)(x)\right| \leq\left(\frac{s}{t}\right)^{\gamma_{k}+\frac{d}{2}}\left(G_{s}^{k}(|f|)\right)(x) .
$$

(iv) If $f \in C_{b}\left(\mathbb{R}^{d}\right)$, then $G_{t}^{k}(f)(x) \rightarrow f(\xi)$ as $(x, t) \rightarrow(\xi, 0)$.

Proof. (i) We use the estimations (3) for the partial derivatives of $E_{k}$, these provide sufficient decay properties for the derivatives of $\mathcal{T}_{-y}^{k} F_{t}^{k}$, allowing the necessary differentiations of $G_{t}^{k}(f)$ under the integral sign by using dominated convergence Theorem.

From Fubini's Theorem, Proposition 3.2 of [14] and the following relation

$$
\int_{\mathbb{R}^{d}} F_{t}^{k}(y) \mathcal{T}_{-y}^{k}\left(\mathcal{T}_{-z}^{k} F_{s}^{k}\right)(x) h_{k}^{2}(y) d y=F_{t}^{k} *_{k} \mathcal{T}_{-z}^{k} F_{s}^{k}(x)=\mathcal{T}_{-z}^{k} F_{s+t}^{k}(x), z, x \in \mathbb{R}^{d},
$$

we deduce (ii).

(iii) We have, for all $z \in \mathbb{R}^{d}$ and $0<t \leq s$,

$$
F_{t}^{k}(z) \leq\left(\frac{s}{t}\right)^{\gamma_{k}+\frac{d}{2}} F_{s}^{k}(z)
$$

Then we obtain the result using Proposition 1.2. The assertion (iv) is proved in [9] p. 538 .

REMARK. The assertion (ii) is true when we take $f \in L^{p}\left(\mathbb{R}^{d}, h_{k}^{2}\right), p \in[1,+\infty]$.

Theorem 2.4. Let $p \in[1,+\infty]$, and let $f \in L^{p}\left(\mathbb{R}^{d}, h_{k}^{2}\right)$. Then, the $k$-heat transform $G_{t}^{k}(f)$ of $f$ has the following properties:

(i) For all $t>0$ and $m \in \mathbb{N}$, we have

$$
\left\|G_{t}^{k}(f)\right\|_{k, p} \leq c_{h}^{-1}\|f\|_{k, p}, \quad\left\|\partial_{t}^{m} G_{t}^{k}(f)\right\|_{k, p} \leq B(d, k, m) t^{-m}\|f\|_{k, p},
$$

where $B(d, k, m)$ is a constant.

(ii) Let $G=\mathbf{Z}_{2}^{d}$, if $1 \leq p<r<\infty$ and $\delta=\frac{1}{p}-\frac{1}{r}$, then for all $t>0$

$$
\left\|G_{t}^{k}(f)\right\|_{k, r} \leq t^{-\left(\gamma_{k}+\frac{d}{2}\right) \delta} c_{h}^{\delta-2}\|f\|_{k, p}
$$

and $\left\|G_{t}^{k}(f)\right\|_{k, r}=o\left(t^{-\left(\gamma_{k}+\frac{d}{2}\right) \delta}\right)$, as $t \rightarrow 0^{+}$.

Further, if $\nu \in \mathbb{N}^{d}$ and $m \in \mathbb{N}$, then for all $t>0$ we have

$$
\left\|\mathcal{D}^{\nu} G_{t}^{k}(f)\right\|_{k, r} \leq B(d, k, \nu, r, p) t^{-\frac{|\nu|}{2}-\left(\gamma_{k}+\frac{d}{2}\right) \delta}\|f\|_{k, p},
$$




$$
\left\|\partial_{t}^{m} G_{t}^{k}(f)\right\|_{k, r} \leq B(d, k, m, r, p) t^{-m-\left(\gamma_{k}+\frac{d}{2}\right) \delta}\|f\|_{k, p},
$$

where $B(d, k, \nu, r, p)$ and $B(d, k, m, r, p)$ are constants. In particular, $G_{t}^{k}(f), \mathcal{D}^{\nu} G_{t}^{k}(f)$ and $\partial_{t}^{m} G_{t}^{k}(f)$ are bounded on $\mathbb{R}^{d} \times[c,+\infty[$ for each $c>0$.

(iii) For each $t>0$ and $m \in \mathbb{N}$, the functions $x \mapsto G_{t}^{k}(f)(x)$ and $x \mapsto \partial_{t}^{m} G_{t}^{k}(f)(x)$ are uniformly continuous on $\mathbb{R}^{d}$.

(iv) For $f \in L^{p}\left(\mathbb{R}^{d}, h_{k}^{2}\right), 1 \leq p<\infty$ or $f \in C_{0}\left(\mathbb{R}^{d}\right), p=\infty$, then $\lim _{t \rightarrow 0} \| G_{t}^{k}(f)-$ $f \|_{k, p}=0$.

(v) $t \mapsto\left\|G_{t}^{k}(f)\right\|_{k, p}$ is continuous on $] 0,+\infty[$.

(vi) Let $G=\mathbf{Z}_{2}^{d}$, if $1 \leq p<\infty$ and $m$ is a positive integer, then $\left\|\partial_{t}^{m} G_{t}^{k}(f)\right\|_{k, p}=$ $o\left(t^{-m}\right)$ as $t \rightarrow 0^{+}$.

Proof. (i) is a consequence from the relation $G_{t}^{k}(f)=F_{t}^{k} *_{k} f$, Theorem 2.3(i), Theorem 1.4 and Lemma 2.1(vi).

(ii) Let $q$ such that $\frac{1}{r}=\frac{1}{p}+\frac{1}{q}-1$. From Theorem 2.3 (ii), we can write $G_{t}^{k}(f)(x)=$ $F_{\frac{t}{2}}^{k} *_{k} G_{\frac{t}{2}}^{k}(f)(x)$. Using Proposition 7.2 of [14] and the result (i), we have

$$
\left\|G_{t}^{k}(f)\right\|_{k, r} \leq c_{h}^{-1}\left\|F_{\frac{t}{2}}^{k}\right\|_{k, q}\|f\|_{k, p} .
$$

By a simple verification, we deduce that $\left\|F_{\frac{t}{2}}^{k}\right\|_{k, q} \leq t^{-\left(\gamma_{k}+\frac{d}{2}\right) \delta} c_{h}^{\delta-1}$. So, we obtain (7) and in the same way we obtain (8) and (9).

(iii) From Theorem 2.3(ii), we have for all $t>0$

$$
\left|G_{t}^{k}(f)(x)-G_{t}^{k}(f)\left(x^{\prime}\right)\right| \leq\left\|G_{\frac{t}{2}}^{k}(f)\right\|_{\infty}\left\|\mathcal{T}_{x}^{k} F_{\frac{t}{2}}^{k}-\mathcal{T}_{x^{\prime}}^{k} F_{\frac{t}{2}}^{k}\right\|_{k, 1} .
$$

Taking limit as $x$ goes to $x^{\prime}$ and using dominated convergence theorem, we deduce the result. Analogous reasoning proves that the function $x \mapsto \partial_{t}^{m} G_{t}^{k}(f)(x)$ is uniformly continous on $\mathbb{R}^{d}$. Since $G_{t}^{k}(f)=F_{t}^{k} *_{k} f$, using Theoerm 4.2 [14], we obtain (iv).

(v) Suppose first that $p \in\left[1,+\infty\left[\right.\right.$. Since we have $\left|G_{t}^{k}(f)(x)\right| \leq 2^{\gamma_{k}+\frac{d}{2}} G_{s}^{k}(|f|)(x)$, whenever $0<\frac{1}{2} s \leq t<s$; also $\left\|G_{s}^{k}(|f|)\right\|_{k, p} \leq\|f\|_{k, p}$ and $t \mapsto\left|G_{t}^{k}(f)\right|^{p}$ is continuous, an application of dominated convergence Theorem shows that the function $t \mapsto\left\|G_{t}^{k}(f)\right\|_{k, p}$ is continuous on $\left[\frac{1}{2} s, s\right]$ for each $s>0$, and therefore is continuous on ] $0,+\infty[$.

If $p=\infty$, then by applying the relation (6), Proposition 1.2 and the following relation

$$
\int_{\mathbb{R}^{d}} \mathcal{T}_{x}^{k} F_{t}^{k}(y) h_{k}^{2}(y) d y=\int_{\mathbb{R}^{d}} F_{t}^{k}(y) h_{k}^{2}(y) d y,
$$

we obtain for $0<s<t$

$$
0 \leq\left\|G_{t}^{k}(f)\right\|_{\infty}-\left\|G_{s}^{k}(f)\right\|_{\infty} \leq c_{h}^{-1}\|f\|_{\infty}\left|\left(\frac{t}{s}\right)^{\gamma_{k}+\frac{d}{2}}-1\right| .
$$

The result is shown to hold by a limiting argument.

(vi) By using Lemma 2.1(v), Theorem 2.3(i) and Minkowski's inequality, we obtain

$$
\left\|\partial_{t}^{m} G_{t}^{k}(f)\right\|_{k, p} \leq \int_{\mathbb{R}^{d}}\left\|\mathcal{T}_{-y}^{k} f-f\right\|_{k, p}\left|\partial_{t}^{m} F_{t}^{k}(y)\right| h_{k}^{2}(y) d y .
$$

Since $\lim _{y \rightarrow 0}\left\|\mathcal{T}_{-y}^{k} f-f\right\|_{k, p}=0$, then for each $\epsilon$, we can find $\beta>0$ such that $\| \mathcal{T}_{-y}^{k} f-$ $f \|_{k, p} \leq \epsilon$, for $\|y\|<\beta$ and we also have $\left\|\mathcal{T}_{-y}^{k} f-f\right\|_{k, p} \leq 4\|f\|_{k, p}$. Thus

$$
\left\|\partial_{t}^{m} G_{t}^{k}(f)\right\|_{k, p} \leq \epsilon \int_{\mathbb{R}^{d}}\left|\partial_{t}^{m} F_{t}^{k}(y)\right| h_{k}^{2}(y) d y+4\|f\|_{k, p} \int_{\|y\| \geq \beta}\left|\partial_{t}^{m} F_{t}^{k}(y)\right| h_{k}^{2}(y) d y .
$$


The first integral on the right does not exceed $B(d, k, m) t^{-m}$, and the second integral on the right is $o\left(t^{-m}\right)$. This completes the proof.

3. Dunkl-Bessel potential. This section is devoted to the study of the DunklBessel potential of a function, then of a $k$-temperature and finally of a tempered distribution.

Definition 3.1. For any $f \in L^{p}\left(\mathbb{R}^{d}, h_{k}^{2}\right)$, where $1 \leq p \leq \infty$, and for any $\alpha>0$, the $k$-Bessel potential $\mathcal{J}_{\alpha}^{k} f$ of order $\alpha$ of $f$ is given by

$$
\mathcal{J}_{\alpha}^{k} f=b_{\alpha}^{k} *_{k} f
$$

with the kernel function

$$
\begin{aligned}
b_{\alpha}^{k}(x) & =\frac{1}{2^{\gamma_{k}+\frac{d}{2}} \Gamma\left(\frac{\alpha}{2}\right)} \int_{0}^{+\infty} e^{-t} e^{-\frac{\|x\|^{2}}{4 t}} t^{-\gamma_{k}+\frac{(\alpha-d)}{2}-1} d t \\
& =\frac{1}{2^{\frac{\alpha}{2}-1} \Gamma\left(\frac{\alpha}{2}\right)}\|x\|^{\frac{1}{2}(\alpha-d)-\gamma_{k}} K_{\frac{\alpha}{2}-\frac{d}{2}-\gamma_{k}}(\|x\|) .
\end{aligned}
$$

Here

$$
K_{\beta}(z)=\frac{\pi}{2}\left\{\frac{J_{-\beta}(z)-J_{\beta}(z)}{\sin \beta \pi}\right\}
$$

where $J_{\beta}$ is the modified Bessel function of the first kind with series expansion

$$
J_{\beta}(z)=\sum_{n=0}^{+\infty} \frac{\left(\frac{1}{2} z\right)^{\beta+2 n}}{n ! \Gamma(\beta+n+1)} .
$$

The $k$-Bessel potentials are bounded operators from $L^{p}\left(\mathbb{R}^{p}, h_{k}^{2}\right)$ to itself for $1 \leq p \leq \infty$ (see [15]), i.e. if $f \in L^{p}\left(\mathbb{R}^{d}, h_{k}^{2}\right)$ and $\alpha>0$, then $\mathcal{J}_{\alpha}^{k} f \in L^{p}\left(\mathbb{R}^{d}, h_{k}^{2}\right)$ and $\left\|\mathcal{J}_{\alpha}^{k} f\right\|_{k, p} \leq$ $\|f\|_{k, p}$. Further, for $\alpha, \beta>0$

$$
\mathcal{J}_{\alpha}^{k}\left(\mathcal{J}_{\beta}^{k} f\right)=\mathcal{J}_{\alpha+\beta}^{k} f
$$

Since, $b_{\alpha}^{k} \in \mathcal{E}_{\text {rad }}\left(\mathbb{R}^{d}\right)$ (the subspace of radial functions in $\mathcal{E}\left(\mathbb{R}^{d}\right)$ ). Then according to Proposition 1.2, relation (1) and formula (17) given in [2] p.313, we have

$$
\begin{gathered}
\mathcal{T}_{y}^{k} b_{\alpha}^{k}(x)=\frac{1}{2^{\frac{\alpha}{2}-1} \Gamma\left(\frac{\alpha}{2}\right)} \int_{C(y)}\left(\|x\|^{2}+\|y\|^{2}+2<x, \xi>\right)^{\frac{1}{2}\left(\frac{\alpha-d}{2}-\gamma_{k}\right)} \times \\
K_{\frac{\alpha}{2}-\gamma_{k}-\frac{d}{2}}\left(\sqrt{\|x\|^{2}+\|y\|^{2}+2<x, \xi>}\right) d \mu_{y}^{k}(\xi) .
\end{gathered}
$$

Our discussion of $k$-Bessel potential is motivated by the following theorem.

Theorem 3.2. Let $\alpha>0,1 \leq p \leq \infty$ and let $f \in L^{p}\left(\mathbb{R}^{p}, h_{k}^{2}\right)$, then (i) The $k$-Bessel potential $\mathcal{J}_{\alpha}^{k} f$ of order $\alpha$ of $f$ is given for almost all $x$ by

$$
\mathcal{J}_{\alpha}^{k} f(x)=\frac{1}{\Gamma\left(\frac{\alpha}{2}\right)} \int_{0}^{+\infty} t^{\frac{\alpha}{2}-1} e^{-t} G_{t}^{k}(f)(x) d t,
$$


where $G_{t}^{k}(f), t>0$, is the $k$-heat transform of $f$ on $\mathbb{R}^{d}$.

(ii) The $k$-heat transform of $\mathcal{J}_{\alpha}^{k} f, \alpha>0$, on $\mathbb{R}^{d}$ is the function $G_{s}^{k}\left(\mathcal{J}_{\alpha}^{k} f\right)$ given by

$$
G_{s}^{k}\left(\mathcal{J}_{\alpha}^{k} f\right)(x)=\frac{1}{\Gamma\left(\frac{\alpha}{2}\right)} \int_{0}^{+\infty} t^{\frac{\alpha}{2}-1} e^{-t} G_{s+t}^{k}(f)(x) d t .
$$

Moreover, for each $s>0$, the function $x \mapsto G_{s}^{k}\left(\mathcal{J}_{\alpha}^{k} f\right)(x)$ is the $k$-Bessel potential of $x \mapsto G_{s}^{k}(f)(x)$.

Proof. Let $g: \mathbb{R}^{d} \rightarrow \overline{\mathbb{R}}$ be given by

$$
g(x)=\frac{1}{\Gamma\left(\frac{\alpha}{2}\right)} \int_{0}^{+\infty} t^{\frac{\alpha}{2}-1} e^{-t}\left|G_{t}^{k}(f)(x)\right| d t .
$$

By Minkowski's inequality and Theorem 2.4(i), we have $\|g\|_{k, p} \leq c_{h}^{-1}\|f\|_{k, p}$. Hence, the integral on the right of (12) exists a.e.

We may assume without loss of generality that $f \geq 0$. From Fubini-Tonelli's theorem and Lemma 2.1(viii), we have

$$
\begin{gathered}
\int_{0}^{+\infty} t^{\frac{\alpha}{2}-1} e^{-t} G_{t}^{k}(f)(x) d t= \\
2^{-\gamma_{k}-\frac{d}{2}} \int_{\mathbb{R}^{d}} f(y)\left\{\int_{0}^{+\infty} t^{\frac{\alpha}{2}-\gamma_{k}-\frac{d}{2}-1} e^{-t-\frac{\|x\|^{2}+\|y\|^{2}}{4} t^{-1}} E_{k}\left(x, \frac{y}{2 t}\right) d t\right\} h_{k}^{2}(y) d y .
\end{gathered}
$$

Using the relation (2), Fubini-Tonelli's theorem and formula (17) given in [2] p.313, we obtain

$$
\begin{gathered}
\int_{0}^{+\infty} t^{\frac{\alpha}{2}-\gamma_{k}-\frac{d}{2}-1} e^{-t-\frac{\|x\|^{2}+\|y\|^{2}}{4} t^{-1}} E_{k}\left(\frac{x}{2 t}, y\right) d t=2^{-\frac{\alpha}{2}+\gamma_{k}+\frac{d}{2}+1} \times \\
\int_{C(y)}\left(\|x\|^{2}+\|y\|^{2}-2<\xi, x>\right)^{\frac{1}{2}\left(\frac{\alpha-d}{2}-\gamma_{k}\right)} K_{\frac{\alpha}{2}-\gamma_{k}-\frac{d}{2}}\left(\sqrt{\|x\|^{2}+\|y\|^{2}-2<\xi, x>}\right) d \mu_{y}^{k}(\xi) .
\end{gathered}
$$

Then (12) is a consequence of (11). We deduce (13) from the assertion (i), Fubini's theorem and Theorem 2.3(ii).

Definition 3.3. A function $U^{k}$ on $\left.\Omega=\mathbb{R}^{d} \times\right] 0,+\infty[$ is said to be a $k$-temperature if it is indefinitely differentiable on $\Omega$ and satisfies at each point of $\Omega$ the heat equation for the $k$-Laplacian i.e.

$$
\triangle_{k, x} U^{k}(x, t)=\partial_{t} U^{k}(x, t)
$$

We refer to [8] for the following definition of a $k$-Bessel potential for certain $k$ temperatures.

Definition 3.4. Let $\mathcal{T}^{k}(\Omega)$ denote the linear space of $k$-temperatures $U^{k}$ on $\Omega$ with the properties that if $(\nu, m) \in \mathbb{N}^{d} \times \mathbb{N}, b>0, c>0$, and $S$ is a compact subset of $\mathbb{R}^{d}$, there exists $C \geq 0$ such that

$$
\left|\mathcal{D}^{\nu} \partial_{t}^{m} U^{k}(x, t)\right| \leq C t^{-b} e^{t}, \text { for all }(x, t) \in S \times[c,+\infty[\text {. }
$$


For any real number $\alpha$ and for any $U^{k} \in \mathcal{T}^{k}(\Omega)$ we define $\mathcal{J}_{\alpha}^{k}\left(U^{k}\right)$ to be the function with domain $\Omega$ given as follows:

(i) $\mathcal{J}_{0}^{k}\left(U^{k}\right)=U^{k}$;

(ii) if $\alpha>0$, then

$$
\mathcal{J}_{\alpha}^{k}\left(U^{k}\right)(x, s)=\frac{1}{\Gamma\left(\frac{\alpha}{2}\right)} \int_{0}^{+\infty} t^{\frac{\alpha}{2}-1} e^{-t} U^{k}(x, s+t) d t
$$

(iii) if $\alpha$ is a negative even integer, say $\alpha=-2 m$, then

$$
\mathcal{J}_{\alpha}^{k}\left(U^{k}\right)(x, s)=\mathcal{J}_{-2 m}^{k}\left(U^{k}\right)(x, s)=(-1)^{m} e^{s} \partial_{s}^{m}\left\{e^{-s} U^{k}(x, s)\right\} ;
$$

(iv) if $\alpha=-\beta<0$ and $\beta$ is not an even integer, then

$$
\mathcal{J}_{\alpha}^{k}\left(U^{k}\right)=\mathcal{J}_{-\beta}^{k}\left(U^{k}\right)=\mathcal{J}_{2 m-\beta}^{k}\left(\mathcal{J}_{-2 m}^{k}\left(U^{k}\right)\right) ;
$$

where $m=\left[\frac{1}{2} \beta\right]+1^{1}$, and where $\mathcal{J}_{2 m-\beta}^{k}$ and $\mathcal{J}_{-2 m}^{k}$ are defined as in (ii) and (iii).

The proof of the following theorem follows the argument for the ordinary case (i.e., $k=0$ ) as given in [8] p.401.

Theorem 3.5. Let $U^{k} \in \mathcal{T}^{k}(\Omega)$, and let $\mathcal{J}_{\alpha}^{k}\left(U^{k}\right)$ be defined as in definition 3.4. Then

(i) for each real $\alpha$ the function $\mathcal{J}_{\alpha}^{k}\left(U^{k}\right) \in \mathcal{T}^{k}(\Omega)$,

(ii) for all real $\alpha, \beta$

$$
\mathcal{J}_{\alpha}^{k}\left(\mathcal{J}_{\beta}^{k}\left(U^{k}\right)\right)=\mathcal{J}_{\alpha+\beta}^{k}\left(U^{k}\right)
$$

Conollany 3.6. For each real number $\alpha, \mathcal{J}_{\alpha}^{k}$ is a linear isomorphism of $\mathcal{T}^{k}(\Omega)$ onto itself, with inverse $\mathcal{J}_{-\alpha}^{k}$.

Definition 3.7. Let $T$ be in $S^{\prime}\left(\mathbb{R}^{d}\right)$ and $\varphi$ in $S\left(\mathbb{R}^{d}\right)$. The Dunkl convolution product of $T$ and $\varphi$ is the function $T *_{k} \varphi$ defined by

$$
\forall x \in \mathbb{R}^{d}, T *_{k} \varphi(x)=<T_{y}, \mathcal{T}_{-x}^{k} \check{\varphi}(y)>.
$$

The Dunkl transform of a distribution $T$ in $S^{\prime}\left(\mathbb{R}^{d}\right)$ is defined by

$$
<\mathcal{F}_{k}(T), \varphi>=<T, \mathcal{F}_{k}(\varphi)>, \varphi \in S\left(\mathbb{R}^{d}\right) .
$$

Proposition 3.8. ([18]) (i) For $T$ in $S^{\prime}\left(\mathbb{R}^{d}\right)$ and $\varphi$ in $S\left(\mathbb{R}^{d}\right)$ the function $T *_{k} \varphi$ belongs to $\mathcal{E}\left(\mathbb{R}^{d}\right)$ and we have

$$
\mathcal{D}^{\nu}\left(T *_{k} \varphi\right)=T *_{k} \mathcal{D}^{\nu} \varphi
$$

(ii) Let $T$ be in $S^{\prime}\left(\mathbb{R}^{d}\right)$ and $\varphi$ in $S\left(\mathbb{R}^{d}\right)$. Then the distribution on $\mathbb{R}^{d}$ given by $T *_{k} \varphi$ belongs to $S^{\prime}\left(\mathbb{R}^{d}\right)$ and we have

$$
\mathcal{F}_{k}\left(T *_{k} \varphi\right)=\mathcal{F}_{k}(\varphi) \mathcal{F}_{k}(T)
$$

\footnotetext{
${ }^{1}$ Here $[x]$ denotes the greatest integer not exceeding $x, x \in \mathbb{R}$.
} 
Remarks. If $T, g \in S^{\prime}\left(\mathbb{R}^{d}\right)$ and $\mathcal{F}_{k}(g) \in \mathcal{O}$ (the class of indefinitely differentiable functions on $\mathbb{R}^{d}$ all of whose Dunkl derivatives are slowly increasing), then $T *_{k} g$ is the element of $S^{\prime}\left(\mathbb{R}^{d}\right)$ given by $\mathcal{F}_{k}\left(T *_{k} g\right)=\mathcal{F}_{k}(T) \mathcal{F}_{k}(g)$.

Definition 3.9. For any $T \in S^{\prime}\left(\mathbb{R}^{d}\right)$, the $k$-heat transform of $T$ is given by

$$
G_{t}^{k}(T)(x)=T *_{k} F_{t}^{k}(x) .
$$

Lemma 3.10. Let $T \in S^{\prime}\left(\mathbb{R}^{d}\right)$, and let $G_{t}^{k}(T)$ be its $k$-heat transform. Then $G_{t}^{k}(T)$ is a k-temperature on $\Omega$. Moreover, for each $(\nu, m) \in \mathbb{N}^{d} \times \mathbb{N}, c>0$, there exist non-negative numbers $a, b, r$ and $C$ such that for all $(x, t) \in \mathbb{R}^{d} \times[c,+\infty[$

$$
\left|\mathcal{D}^{\nu} \partial_{t}^{m} G_{t}^{k}(T)(x)\right| \leq C\left(1+\|x\|^{2}\right)^{a}(1+t)^{b} t^{-r} .
$$

Proof. Since $F_{t}^{k}$ is a $k$-temperature and $T$ is linear, then Proposition 3.8 gives that $G_{t}^{k}(T)$ is a $k$-temperature on $\Omega$.

As $T$ belongs to $S^{\prime}\left(\mathbb{R}^{d}\right)$, then there exist a positive constant $c_{0}$ and $s_{0}, l_{0} \in \mathbb{N}$ such that

$$
\left|T *_{k} \varphi(x)\right| \leq c_{0} \rho_{s_{0}, l_{0}}\left(\mathcal{T}_{x}^{k} \varphi\right), \forall \varphi \in S\left(\mathbb{R}^{d}\right)
$$

In particular,

$$
\left|\mathcal{D}^{\nu} \partial_{t}^{m} G_{t}^{k}(T)(x)\right| \leq c_{0} \rho_{s_{0}, l_{0}}\left(\mathcal{T}_{x}^{k} \mathcal{D}^{\nu} \partial_{t}^{m} F_{t}^{k}\right)
$$

But using the inequality

$$
\forall x, y \in \mathbb{R}^{d}, 1+\|x+y\|^{2} \leq 2\left(1+\|x\|^{2}\right)\left(1+\|y\|^{2}\right),
$$

the relations (4), (1) and the representation of $V_{k}^{-1}$ (see [16]), we deduce that there exist a positive constant $c_{1}$ and $s, l \in \mathbb{N}$ such that

$$
\rho_{s_{0}, l_{0}}\left(\mathcal{T}_{x}^{k} \mathcal{D}^{\nu} \partial_{t}^{m} F_{t}^{k}\right) \leq c_{1}\left(1+\|x\|^{2}\right)^{l_{0}} \rho_{s, l}\left(\mathcal{D}^{\nu} \partial_{t}^{m} F_{t}^{k}\right) .
$$

Further, by Lemma 2.1(ii)(iii), we have for all $(y, t) \in \mathbb{R}^{d} \times\left[c,+\infty\left[, \lambda \in \mathbb{N}^{d}\right.\right.$

$$
\begin{aligned}
& \left|\mathcal{D}^{\lambda} \partial_{t}^{m} F_{t}^{k}(y)\right| \\
\leq & B(d, \lambda, k, m) t^{-m-\gamma_{k}-\frac{d}{2}}\left[t^{-|\lambda|}\left(1+\frac{\|y\|}{\sqrt{t}}\right)^{2 m}+t^{-\frac{|\lambda|}{2}}\left(1+\|y\| t^{-\frac{1}{2}}\right)^{|\lambda|}\right] e^{-\frac{\|y\|^{2}}{4} t^{-1}} .
\end{aligned}
$$

The elementary inequality $(\sqrt{t}+\|y\|)^{p} \leq 2^{\frac{1}{2} p}(1+t)^{\frac{1}{2} p}\left(1+\|y\|^{2}\right)^{\frac{1}{2} p}, \quad p \in \mathbb{N}$, leads to

$$
\left|\mathcal{D}^{\lambda} \partial_{t}^{m} F_{t}^{k}(y)\right| \leq B(d, \lambda, k, m, c) t^{-m-|\lambda|-\gamma_{k}-\frac{d}{2}}(1+t)^{m+\frac{|\lambda|}{2}}\left(1+\|y\|^{2}\right)^{m+\frac{|\lambda|}{2}} e^{-\frac{\|y\|^{2}}{4 t}} .
$$

Hence, there exists $m_{0} \in \mathbb{N}$ such that

$$
\begin{aligned}
& \rho_{s, l}\left(\mathcal{D}^{\nu} \partial_{t}^{m} F_{t}^{k}\right) \\
\leq & \tilde{B}(d, k, \nu, l, m, c) t^{-m-|\nu|-m_{0}-\gamma_{k}-\frac{d}{2}}(1+t)^{m+\frac{|\nu|}{2}+\frac{m_{0}}{2}} \sup _{y \in \mathbb{R}^{d}}\left\{\left(1+\|y\|^{2}\right)^{m+\frac{|\nu|}{2}+l+s} e^{-\frac{\|y\|^{2}}{4 t}}\right\} .
\end{aligned}
$$

Since

$$
\sup _{\rho \geq 0}(1+\rho)^{m+\frac{|\nu|}{2}+l+s} e^{-\frac{\rho}{4 t}} \leq B(\nu, m)(1+t)^{m+\frac{|\nu|}{2}+l+s},
$$


then we have

$$
\left|\mathcal{D}^{\nu} \partial_{t}^{m} G_{t}^{k}(T)(x)\right| \leq C\left(1+\|x\|^{2}\right)^{l_{0}} t^{-m-|\nu|-m_{0}-\gamma_{k}-\frac{d}{2}}(1+t)^{2 m+|\nu|+l+s+\frac{m_{0}}{2}} .
$$

Definition 3.11. For any real number $\alpha$ and for any $T \in S^{\prime}\left(\mathbb{R}^{d}\right)$ the $k$-Bessel potential of order $\alpha$ of $T$ is the element $\mathcal{J}_{\alpha}^{k}(T)$ of $S^{\prime}\left(\mathbb{R}^{d}\right)$ given by the relation

$$
\mathcal{F}_{k}\left(\mathcal{J}_{\alpha}^{k}(T)\right)=\left(1+\|\cdot\|^{2}\right)^{-\frac{\alpha}{2}} \mathcal{F}_{k}(T),
$$

where the identity is to be understood in the sense of distributions.

REMARK.

- For all real $\alpha, \beta$, and all $T \in S^{\prime}\left(\mathbb{R}^{d}\right)$, we have $\mathcal{J}_{\alpha}^{k}\left(\mathcal{J}_{\beta}^{k}(T)\right)=\mathcal{J}_{\alpha+\beta}^{k}(T)$.

- By definition, we have

$$
\mathcal{J}_{\alpha}^{k}(T)=T *_{k} b_{\alpha}^{k},
$$

where $b_{\alpha}^{k}$ is a tempered distribution whose Dunkl transform $\mathcal{F}_{k}\left(b_{\alpha}^{k}\right)=[(1+$ $\left.\left.\|\cdot\|^{2}\right)^{-\frac{\alpha}{2}}\right],{ }^{2}$.

- If $f \in L^{p}\left(\mathbb{R}^{d}, h_{k}^{2}\right)$, where $1 \leq p \leq \infty$ and $\alpha>0$, then

$$
\mathcal{J}_{\alpha}^{k}([f])=\mathcal{J}_{\alpha}^{k}(f)=f *_{k} b_{\alpha}^{k} .
$$

Using the following formula

$$
K_{\nu}(z) \sim\left(\frac{\pi}{2 z}\right)^{\frac{1}{2}} e^{-z}, \text { as } z \rightarrow \infty, \text { for all } \nu \in \mathbb{R},
$$

we deduce that, if $\alpha>0$ and $y$ be fixed in $\mathbb{R}^{d}$

$$
\mathcal{T}_{y}^{k} b_{\alpha}^{k}(x) \sim B(d, \alpha, k)\|x\|^{\frac{1}{2}(\alpha-d-1)-\gamma_{k}} e^{-\|x\|} \text {, as }\|x\| \rightarrow \infty .
$$

Theorem 3.12. Let $\alpha \in \mathbb{R}$, and $T \in S^{\prime}\left(\mathbb{R}^{d}\right)$. Then (i) $G_{t}^{k}(T) \in \mathcal{T}^{k}(\Omega)$, and $\mathcal{J}_{\alpha}^{k}\left(G_{t}^{k}(T)\right)$ is the k-heat transform of $\mathcal{J}_{\alpha}^{k}(T)$.

(ii) For each $t>0$, the function $x \mapsto \mathcal{J}_{\alpha}^{k}\left(G_{t}^{k}(T)\right)(x)$ is the $k$-Bessel potential of order $\alpha$ of $x \mapsto G_{t}^{k}(T)(x)$, in the distributional sense.

Proof. (i) By Lemma 3.10, we deduce that $(x, t) \mapsto G_{t}^{k}(T)(x)$ belongs to $\mathcal{T}^{k}(\Omega)$ and $x \mapsto G_{t}^{k}(T)(x)$ belongs to $\mathcal{O}$, for each $t>0$. So that $\mathcal{J}_{\alpha}^{k}\left(G_{t}^{k}(T)\right)(x, t)$ and $\mathcal{J}_{\alpha}^{k}\left(G_{t}^{k}(T)\right)$ are defined. It is easily verified that

$$
G_{t}^{k}\left(\mathcal{J}_{\alpha}^{k}(T)\right)=\mathcal{J}_{\alpha}^{k}\left(G_{t}^{k}(T)\right)
$$

in the distributional sense.

(ii) By the definition 3.9 and Lemma 2.1(ix), we obtain

$$
G_{t}^{k}\left(G_{s}^{k}(T)\right)=G_{t+s}^{k}(T), \text { for } s, t>0
$$

\footnotetext{
${ }^{2}[f]$ is the distribution on $\mathbb{R}^{d}$ associated with the function $f$. In addition $[f]$ belongs to $S^{\prime}\left(\mathbb{R}^{d}\right)$, when $f \in L^{p}\left(\mathbb{R}^{d}, h_{k}^{2}\right)$ or $f$ is slowly increasing.
} 
Suppose first $\alpha>0$. Since $G_{t}^{k}(T) \in \mathcal{O}, t>0$, then from the relation (18), we have

$$
G_{t}^{k}\left(\mathcal{J}_{\alpha}^{k}(T)\right)(x)=\int_{\mathbb{R}^{d}} \mathcal{T}_{-y}^{k} b_{\alpha}^{k}(x) G_{t}^{k}(T)(y) d y .
$$

From the relation (19), we remark that the integral on the right is finite. In view of relations (11), (15), (14) and (20) we obtain

$$
G_{t}^{k}\left(\mathcal{J}_{\alpha}^{k} T\right)(x)=\frac{1}{\Gamma\left(\frac{\alpha}{2}\right)} \int_{0}^{+\infty} s^{\frac{\alpha}{2}-1} e^{-s} G_{t+s}^{k}(T)(x) d s=\mathcal{J}_{\alpha}^{k}\left(G_{t}^{k}(T)\right)(x, t) .
$$

Next, let $\alpha=-\beta<0$, we have

$$
\mathcal{J}_{\beta}^{k}\left(G_{t}^{k}\left(\mathcal{J}_{-\beta}^{k}(T)\right)\right)=G_{t}^{k}(T)
$$

Hence, by Theorem 3.5(ii), we deduce

$$
\mathcal{J}_{-\beta}^{k}\left(G_{t}^{k}(T)\right)(x, t)=G_{t}^{k}\left(\mathcal{J}_{-\beta}^{k}(T)\right)(x) .
$$

4. Dunkl-Flett potential and Dunkl weighted wavelet-like transform associated to the $k$-Poisson transform. We begin this section by introducing the k-Poisson transform which permits to define and study the Dunkl-Flett potential and its relation with the Dunkl weighted wavelet-like transform which is also studied here and we give an associated inversion formula. $\mathbb{R}^{d}$ by

By analogy with the euclidean case [12], we define for $t>0$, the function $P_{t}^{k}$ on

$$
P_{t}^{k}(x):=c_{d, k} \frac{t}{\left(t^{2}+\|x\|^{2}\right)^{\gamma_{k}+\frac{d+1}{2}}}
$$

where $c_{d, k}=\frac{2^{\gamma_{k}+\frac{d}{2}}}{\Gamma\left(\frac{1}{2}\right)} \Gamma\left(\gamma_{k}+\frac{d+1}{2}\right)$.

The function $P_{t}^{k}$, may be called $k$-Poisson kernel. This function has the following basic properties which can be easily verified :

(i) $\mathcal{F}_{k}\left(P_{t}^{k}\right)(x)=e^{-t\|x\|}$, for all $t>0$.

(ii) $\int_{\mathbb{R}^{d}} P_{t}^{k}(y) h_{k}^{2}(y) d y=1$.

(iii) If $\eta>0$, then $\int_{\|y\| \geq \eta} P_{t}^{k}(y) h_{k}^{2}(y) d y \rightarrow 0$, as $t \rightarrow 0$.

However, for $t>0$ and for all $f \in L^{p}\left(\mathbb{R}^{d}, h_{k}^{2}\right), p \in[1, \infty]$, we put

$$
P_{t}^{k} f(x):=P_{t}^{k} *_{k} f(x), x \in \mathbb{R}^{d} .
$$

The function $P_{t}^{k}(f)$ is called the $k$-Poisson transform of $f$.

Before, asserting some properties of the $k$-Poisson transform in a technical lemma we recall that the homologous of maximal function in the Dunkl setting is defined in [14] to be the operator $M_{k}$ acting on $L^{p}\left(\mathbb{R}^{d}, h_{k}^{2}\right)$, given by

$$
M_{k} f(x)=\sup _{r>0} \frac{1}{d_{k} r^{d+2 \gamma_{k}}}\left|f *_{k} \chi_{B_{r}}(x)\right|,
$$

where $\chi_{B_{r}}$ is the characteristic function of the ball $B_{r}$ of radius $r$ centered at 0 and

$$
d_{k}^{-1}=\int_{B_{1}} h_{k}^{2}(y) d y=\left(d+2 \gamma_{k}\right) \int_{S^{d-1}} h_{k}^{2}(x) d \omega(x) .
$$


The maximal function can also be written as

$$
M_{k} f(x)=\sup _{r>0} \frac{\left|\int_{B_{r}} \mathcal{T}_{y}^{k} f(x) h_{k}^{2}(y) d y\right|}{\int_{B_{r}} h_{k}^{2}(y) d y} .
$$

Lemma 4.1. Let $f \in L^{p}\left(\mathbb{R}^{d}, h_{k}^{2}\right), 1 \leq p \leq \infty$ and $P_{t}^{k} f$ is the $k$-Poisson transform of $f$. Then

(a) $\left\|P_{t}^{k} f\right\|_{k, p} \leq\|f\|_{k, p}$;

(b) $\sup _{t>0}\left|P_{t}^{k} f(x)\right| \leq c_{\tilde{d}, k} M_{k} f(x)$, where $c_{\tilde{d}, k}=2^{2 \gamma_{k}+d+1} c_{d, k}$;

(c) $\lim _{t \rightarrow 0} P_{t}^{k} f(x)=f(x)$, where the limit is interpreted in $L_{p, k}$-norm and pointwise a.e.

For $f \in C_{0}\left(\mathbb{R}^{d}\right)$ the convergence is uniform on $\mathbb{R}^{d}$;

(d) $P_{\tau}^{k} P_{t}^{k} f=P_{\tau+t}^{k} f, \tau, t>0$;

(e) $\sup _{x \in \mathbb{R}^{d}}\left|P_{t}^{k} f(x)\right| \leq\left(c_{d, k}\right)^{\frac{1}{p}} t^{\frac{-d-2 \gamma_{k}}{p}}\|f\|_{k, p}, p \in[1,+\infty[$.

Proof. (a) is merely Tonelli's theorem and the fact

$$
\int_{\mathbb{R}^{d}} \mathcal{T}_{x}^{k} P_{t}^{k}(y) h_{k}^{2}(y) d y=\int_{\mathbb{R}^{d}} P_{t}^{k}(y) h_{k}^{2}(y) d y=1
$$

when $p=1$. If $1<p<\infty$, let $q$ be the conjugate exponent to $p$, then using Hölder's inequality, relation (21) and Fubini's theorem we prove the result. When $p=\infty$ the proof is trivial. The assertion (b) and (c) follow from the the writing of $P_{t}^{k} f$ as

$$
P_{t}^{k} f(x)=f *_{k} \psi_{t}^{k}(x)
$$

where $\psi_{t}^{k}$ is the dilation of $\psi^{k}(x)=c_{d, k} \frac{1}{\left(1+\|x\|^{2}\right)^{\gamma_{k}+\frac{d+1}{2}}}$ given by $\psi_{t}^{k}(x)=$ $t^{-2 \gamma_{k}-d} \phi^{k}\left(\frac{x}{t}\right)$, and are the consequences of Theorem 6.2 and Theorem 7.3 [14]. The assertion $(d)$ is clear.

(e) From Proposition 1.2 and relation (1), we have

$$
\mathcal{T}_{x}^{k} P_{t}^{k}(y) \leq c_{d, k} t^{-2 \gamma_{k}-d} .
$$

We deduce the result, using the following inequality

$$
\left|P_{t}^{k} f(x)\right| \leq\left\{\int_{\mathbb{R}^{d}} \mathcal{T}_{x}^{k} P_{t}^{k}(-y)|f(y)|^{p} h_{k}^{2}(y) d y\right\}^{\frac{1}{p}} .
$$

Given a finite Borel measure $\mu$ on $\mathbb{R}$, the notation $\int_{a}^{b} \varphi(t) d \mu(t)$ is used for the integral $\int_{[a, b)} \varphi(t) d \mu(t)$. In case of $\lim _{t \rightarrow a^{+}}|\varphi(t)|=\infty$, we assume that $\mu(\{a\})=0$ and

$$
\int_{[a, b)} \varphi(t) d \mu(t)=\int_{(a, b)} \varphi(t) d \mu(t)
$$

Definition 4.2. For any $f \in L^{p}\left(\mathbb{R}^{d}, h_{k}^{2}\right)$, where $1 \leq p \leq \infty$ and for any $\alpha>0$, the $k$-Flett potential $\mathcal{I}_{k}^{\alpha} f$ of order $\alpha$ of $f$ is given by

$$
\mathcal{I}_{k}^{\alpha} f(x)=\frac{1}{\Gamma(\alpha)} \int_{0}^{+\infty} t^{\alpha-1} e^{-t} P_{t}^{k} f(x) d t .
$$


Remarks. We can also write $\mathcal{I}_{k}^{\alpha} f=\phi_{k}^{\alpha} *_{k} f$, where

$$
\phi_{\alpha}^{k}(x)=\frac{1}{\Gamma(\alpha)} \int_{0}^{+\infty} t^{\alpha-1} e^{-t} P_{t}^{k}(x) d t=\frac{c_{d, k}}{\Gamma(\alpha)}\|x\|^{\alpha-2 \gamma_{k}-d} \int_{0}^{+\infty} \frac{s^{\alpha} e^{-s\|x\|}}{\left(1+s^{2}\right)^{\gamma_{k}+\frac{d+1}{2}}} d s .
$$

It is easily proved from (22) that $\phi_{\alpha}^{k}$ has the following basic properties:

(i) a) If $\alpha>d+2 \gamma_{k}$, then $\phi_{\alpha}^{k}$ is continuous on $\mathbb{R}^{d}$ and $\phi_{\alpha}^{k}(x)>0$, for all $x \in \mathbb{R}^{d}$.

b) If $0<\alpha \leq d+2 \gamma_{k}$, then $\phi_{\alpha}^{k}$ is continuous on $\mathbb{R}^{d} \backslash\{0\}$ and $\phi_{\alpha}^{k}(x)>0$ for all non-zero $x \in \mathbb{R}^{d}$. Further, if $0<\alpha<d+2 \gamma_{k}$, then

$$
\phi_{\alpha}^{k}(x) \sim \frac{c_{d, k} \Gamma\left(\frac{1}{2}(\alpha+1)\right) \Gamma\left(\gamma_{k}+\frac{1}{2}(d-\alpha)\right)}{2 \Gamma(\alpha) \Gamma\left(\gamma_{k}+\frac{1}{2}(d+1)\right)}\|x\|^{\alpha-2 \gamma_{k}-d} \text { as }\|x\| \rightarrow 0,
$$

and

$$
\phi_{d+2 \gamma_{k}}^{k}(x) \sim \frac{c_{d, k}}{2 \Gamma\left(d+2 \gamma_{k}\right)} \log \frac{1}{\|x\|} \text { as }\|x\| \rightarrow 0 .
$$

(ii) $\phi_{\alpha}^{k} \in L^{1}\left(\mathbb{R}^{d}, h_{k}^{2}\right),\left\|\phi_{\alpha}^{k}\right\|_{k, 1}=1$ and $\mathcal{F}_{k}\left(\phi_{\alpha}^{k}\right)(x)=(1+\|x\|)^{-\alpha}$, for all $\alpha>0$.

(iii) $\phi_{\alpha}^{k} *_{k} \phi_{\beta}^{k}=\phi_{\alpha+\beta}^{k}$, whenever $\alpha, \beta>0$.

(iv) $\left\|\mathcal{I}_{k}^{\alpha} f\right\|_{k, p} \leq\|f\|_{k, p}, \forall \alpha>0,1 \leq p \leq \infty$.

(v) For all $\alpha>0, \phi_{\alpha}^{k}(x) \sim \alpha c_{d, k}\|x\|^{-2 \gamma_{k}-d-1}$ as $\|x\| \rightarrow \infty$.

Definition 4.3. A signed Borel measure on $\mathbb{R}_{+}$is said to be a wavelet measure if

$$
\|\mu\| \equiv|\mu|\left(\mathbb{R}_{+}\right) \equiv \int_{\mathbb{R}_{+}} d|\mu|(\eta)<\infty \text { and } \mu\left(\mathbb{R}_{+}\right) \equiv \int_{0}^{\infty} d \mu(\eta)=0 .
$$

DeFINITION 4.4. Let $\mu$ be a wavelet measure on $\mathbb{R}_{+}$. The $k$-weighted wavelet-like transform of $f \in L^{p}\left(\mathbb{R}^{d}, h_{k}^{2}\right)$ associated to the $k$-Poisson transform $P_{t}^{k} f$ and generated by the measure $\mu$ is defined as

$$
\mathcal{W}_{t}^{k, \mu} f(x)=\int_{\mathbb{R}_{+}} e^{-t \eta} P_{t \eta}^{k} f(x) d \mu(\eta), x \in \mathbb{R}^{d}, t>0 .
$$

REMARKS.

- Owing to Lemma 4.1(c), it is assumed that $e^{-t \eta} P_{t \eta}^{k} f(x)_{\mid \eta=0}=f(x)$ and therefore

$$
\int_{\mathbb{R}_{+}} e^{-t \eta} P_{t \eta}^{k} f(x) d \mu(\eta)=\int_{(0, \infty)} e^{-t \eta} P_{t \eta}^{k} f(x) d \mu(\eta)+\mu(\{0\}) f(x) .
$$

- For any fixed $t>0$, the $k$-weighted wavelet-like transforms are bounded operators from $L^{p}\left(\mathbb{R}^{d}, h_{k}^{2}\right)$ to itself for $p \in[1,+\infty]$, i.e., if $f \in L^{p}\left(\mathbb{R}^{d}, h_{k}^{2}\right)$, $t>0,\left\|\mathcal{W}_{t}^{k, \mu} f\right\|_{k, p} \leq\|\mu\|\|f\|_{k, p}$.

The proof of the following Lemma follows the proof of Theorem 3.12 p.60 in [13].

LEMma 4.5. Let $\left\{T_{\epsilon}\right\}, \epsilon>0$, be a family of linear operators mapping $L^{p}\left(\mathbb{R}^{d}, h_{k}^{2}\right)$, $1 \leq p \leq \infty$, into the space of measurable functions on $\mathbb{R}^{d}$. For each $f \in L^{p}\left(\mathbb{R}^{d}, h_{k}^{2}\right)$ define $T^{*} f$ by letting

$$
\left(T^{*} f\right)(x)=\sup _{\epsilon>0}\left|\left(T_{\epsilon} f\right)(x)\right|, x \in \mathbb{R}^{d} .
$$


Suppose that there exists a constant $c>0$ and a real number $q \geq 1$ such that

$$
\int_{\left\{x:\left(T^{*} f\right)(x)>t\right\}} h_{k}^{2}(x) d x \leq\left(\frac{c\|f\|_{k, p}}{t}\right)^{q},
$$

for all $t>0$ and $f \in L^{p}\left(\mathbb{R}^{d}, h_{k}^{2}\right)$. If there exists a dense subset $D$ of $L^{p}\left(\mathbb{R}^{d}, h_{k}^{2}\right)$ such that $\lim _{\epsilon \rightarrow 0}\left(T_{\epsilon} g\right)(x)$ exists and is finite a.e. whenever $g \in D$, then for each $f \in$ $L^{p}\left(\mathbb{R}^{d}, h_{k}^{2}\right), \lim _{\epsilon \rightarrow 0}\left(T_{\epsilon} f\right)(x)$ exists and is finite a.e.

Using the last lemma and lemma 4.1 the proof of the following theorems carried over from classical case setting in [1] without changes.

Theorem 4.6. Let $f \in L^{p}\left(\mathbb{R}^{d}, h_{k}^{2}\right), 1 \leq p<\infty$, or $f \in C_{0}\left(\mathbb{R}^{d}\right)$ when $p=\infty$. Suppose that $\mu$ is a finite Borel measure on $\mathbb{R}_{+}$satisfying

$$
\mu\left(\mathbb{R}_{+}\right) \equiv 0 \text { and } \int_{\mathbb{R}_{+}}|\log \tau| d|\mu|(\tau)<+\infty .
$$

Then

$$
\int_{0}^{+\infty} \mathcal{W}_{t}^{k, \mu} f(x) \frac{d t}{t}=\lim _{\epsilon \rightarrow 0} \int_{\epsilon}^{+\infty} \mathcal{W}_{t}^{k, \mu} f(x) \frac{d t}{t}=c_{\mu} . f(x),
$$

where $c_{\mu}=\int_{0}^{+\infty} \log \frac{1}{\tau} d \mu(\tau)$. The limit in (23) is understood in $L_{p, k}$-norm and pointwise for almost all $x \in \mathbb{R}^{d}$. In case of $f \in C_{0}\left(\mathbb{R}^{d}\right)$, the convergence is uniform on $\mathbb{R}^{d}$.

The next theorem gives a new representation of the $k$-Flett potentials with the aid of the $k$-weighted wavelet-like transform $\mathcal{W}_{t}^{k, \mu}$.

Theorem 4.7. Let $\alpha>0, f \in L^{p}\left(\mathbb{R}^{d}, h_{k}^{2}\right), 1 \leq p \leq \infty$ and let $\mu$ be a finite Borel measure on $\mathbb{R}_{+}$satisfying the conditions

$$
\int_{0}^{+\infty} t^{-\alpha} d|\mu|(t)<\infty \text { and } c_{\alpha, \mu} \equiv \int_{0}^{+\infty} t^{-\alpha} d \mu(t) \neq 0 .
$$

Then

$$
\mathcal{I}_{k}^{\alpha} f(x)=\frac{1}{\Gamma(\alpha) c_{\alpha, \mu}} \int_{0}^{+\infty} t^{\alpha} \mathcal{W}_{t}^{k, \mu} f(x) \frac{d t}{t}
$$

The following theorem gives an inversion formula for the transform $\mathcal{W}_{t}^{k, \mu}$.

Theorem 4.8. Let $\alpha>0, f \in L^{p}\left(\mathbb{R}^{d}, h_{k}^{2}\right), 1 \leq p \leq \infty$ and let $\mu$ be a finite Borel measure on $\mathbb{R}_{+}$satisfying

- $\int_{1}^{\infty} t^{\beta} d|\mu|(t)<\infty$ for some $\beta>\alpha$;

Then

- $\int_{0}^{\infty} t^{k} d \mu(t)=0, k=0,1 \cdots, m$, where $m=[\alpha]$ is the integer part of $\alpha$.

$$
\frac{1}{k_{\alpha, \mu}} \int_{0}^{+\infty} t^{-\alpha} \mathcal{W}_{t}^{k, \mu}\left(\mathcal{I}_{k}^{\alpha} f\right)(x) \frac{d t}{t} \equiv \frac{1}{k_{\alpha, \mu}} \lim _{\epsilon \rightarrow 0} \int_{\epsilon}^{+\infty} t^{-\alpha} \mathcal{W}_{t}^{k, \mu}\left(\mathcal{I}_{k}^{\alpha} f\right)(x) \frac{d t}{t}=f(x),
$$

where

$$
k_{\alpha, \mu}=\left\{\begin{aligned}
\Gamma(-\alpha) \int_{0}^{\infty} t^{\alpha} d \mu(t) & , \quad \text { if } \alpha \in \mathbb{R}_{+} \backslash \mathbb{N} \\
\frac{(-1)^{\alpha+1}}{\alpha !} \int_{0}^{\infty} t^{\alpha} \log t d \mu(t) & , \quad \text { if } \alpha \in \mathbb{N}
\end{aligned}\right.
$$


The limit in (24) is interpreted in $L_{p, k}$-norm and pointwise a.e., for $1 \leq p<\infty$. If $f \in C_{0}\left(\mathbb{R}^{d}\right)$, the convergence is uniform on $\mathbb{R}^{d}$.

\section{REFERENCES}

[1] I. A. Aliev, S. Sezer And M. Eryigit, An Integral Transform Associated to the Poisson Integral and Inversion of Flett Potentials, J. Math. Anal. Appl., 321 (2006), pp. 691-704.

[2] H. Bateman, Tables of Integral Transforms, Vol I, New York (1954).

[3] N. B. Salem and S. Kallel, Mean-Periodic Functions Associated With the Dunkl Operators, Integral Transforms and Special Functions, 15:2 (2004), pp. 155-179.

[4] C. F. DunkL, Differential-difference Operators Associated to Reflection Groups, Trans. Amer. Math. Soc., 311 (1989), pp. 167-183.

[5] C. F. DunkL, Integral Kernels With Reflection Group Invariance, Canad. J. Math., 43 (1991), pp. 1213-1227.

[6] C. F. DunkL, Hankel Transforms Associated to Finite Reflection Groups, contemp. Math., 138 (1992), pp. 123-138.

[7] M. F. E. DE JEU, The Dunkl Transform, Invent. Math., 113 (1993), pp. 147-162.

[8] T. M. Flett, Temperatures, Bessel Potentials And Lipschitz Space, Proc. London Math. Soc., 22 (1971), pp. 385-451.

[9] M. RösLer, Generalized Hermite Polynomials and the Heat Equation For Dunkl Operators, Comm. Math. Phys., 192 (1998), pp. 519-542.

[10] M. Rösler, Positivity of Dunkl's intertwining operator, Duke Math. J., 98 (1999), pp. 445-463.

[11] M. Rösler, A Positive Radial Product Formula For The Dunkl Kernel, Trans. Amer. Math. Soc., 355 (2003), pp. 2413-2438.

[12] E. M. Stein, Singular Integrals and Differentiability Properties of Functions, Princeton University Press, Princeton, (1970).

[13] E. M. Stein and G. Weiss, Introduction to Fourier Analysis on Euclidean Spaces, Princeton University Press, Princeton, NJ, (1971).

[14] S. Thangavelu and Y. Xu, Convolution Operateur and Maximal Function for Dunkl Transform, J. d'Analyse Mathematique, 97 (2005), pp. 25-56.

[15] S. Thangavelu and Y. Xu, Riesz Transform And Riesz Potentials for Dunkl Transform, J. Comp. Appl. Math., to appear.

[16] K. Trimèche, The Dunkl Intertwining Operator on Spaces of Functions and Distributions and Integral Representations of its Dual, Integral transforms and special functions, 12:4 (2001), pp. 349-374.

[17] K. Trimèche, Paley-Wiener Theorems for the Dunkl Transform and Dunkl Translation operators, Integral transforms and special functions, 13 (2002), pp. 17-38.

[18] K. Trimèche, Inversion Formulas for the Dunkl Intertwining Operator and its Dual and Another Proofs of the Geometrical forms of Paley-Wiener Theorems for the Dunkl Transform and, "a personal communication". 